\title{
Maus-tratos infantis e comportamentos sexuais de risco na idade adulta: uma revisão sistemática
}

\author{
Child abuse and sexual risk behavior in adulthood: \\ a systematic review
}

Roberta Hirschmann (http://orcid.org/0000-0002-3775-0310) ${ }^{1}$

Rafaela Costa Martins (https://orcid.org/0000-0003-3538-7228) ${ }^{1}$

Helen Gonçalves (https://orcid.org/0000-0001-6470-3352) ${ }^{1}$

1 Programa de PósGraduação em Epidemiologia, Universidade Federal de Pelotas. R. Marechal Deodoro $1160,3^{\circ}$ andar, Centro. 96020-220 Pelotas RS Brasil.

r.nutri@hotmail.com

\begin{abstract}
Child abuse is a public health problem and can lead to serious consequences, such as involvement in sexual risk behavior (SRB). The scope of this article is to identify the studies that evaluated the association between different types of child maltreatment and SRB in adulthood through a systematic review. The search was conducted in the PubMed, Web of Science, PsycNET and Lilacs databases. The inclusion criteria were: cross-sectional, cohort or case-control design; assessment of individuals who have been exposed to maltreatment before the age of 18 ; evaluation of SRB in adulthood. The search identified 18,951 articles, 34 of which were included in this study. The selection process was performed by two researchers independently. Studies have generally shown that sexual abuse is a risk factor for the tendency of SRB in adulthood, however there is less evidence of this association for other forms of child abuse, such as domestic violence, emotional abuse and neglect. Child maltreatment negatively influences SRB in early adulthood. Further studies are needed to evaluate this relationship longitudinally, including the different types of abuse and the possible mechanisms of this relationship. Key words Sexual behavior, Child abuse, Systematic review
\end{abstract}

Resumo Os maus-tratos infantis são um problema de saúde pública e podem gerar graves consequências, como envolvimento em comportamentos sexuais de risco (CSR). O objetivo deste artigo é identificar os estudos que avaliaram a associação entre diferentes tipos de maus-tratos infantis e CSR na idade adulta, por meio de uma revisão sistemática da literatura. A busca foi realizada nas bases de dados PubMed, Web of Science, PsycNET e Lilacs. Os critérios de inclusão foram: delineamento transversal, coorte ou caso-controle; avaliação de indivíduos que foram expostos a maustratos até os 18 anos e avaliação de CSR na idade adulta. A busca identificou 18.951 artigos, sendo 34 incluídos neste estudo. O processo de seleção foi realizado por duas pesquisadoras de maneira independente. Em geral os estudos mostraram que o abuso sexual é um fator de risco para a adoção de CSR na vida adulta, no entanto há menos evidências dessa associação para as outras formas de maus-tratos, como violência doméstica, abuso emocional e negligência. Os maus-tratos infantis influenciam de maneira negativa os CSR da vida adulta. São necessários novos estudos que avaliem essa relação de maneira longitudinal, incluindo os diferentes tipos de maus-tratos e os possiveis mecanismos dessa relação.

Palavras-chave Comportamento sexual, Maustratos infantis, Revisão sistemática 


\section{Introdução}

Os maus-tratos infantis são definidos como a ocorrência de negligência, abuso físico, emocional ou sexual em menores de 18 anos, podendo gerar inúmeros prejuízos à saúde ${ }^{1}$. Estimativas apontam que os maus-tratos infantis impactam parcela significativa da população mundial: $36,3 \%$ dos adultos no mundo foram vítimas, na infância, de abuso emocional, 22,6\% sofreram abuso físico, $16,3 \%$ relataram negligência física e $12,7 \%$ vivenciaram abuso sexual ${ }^{2}$.

A exposição aos maus-tratos infantis é um problema global de saúde pública, que gera graves consequências ao longo da vida dos indivídu$\mathrm{os}^{1,3}$ e está associada à ocorrência de problemas psicológicos, como transtornos de ansiedade e depressão, bem como à adoção de comportamentos de risco, como abuso de substâncias na adolescência e na idade adulta ${ }^{4-6}$. Mais recentemente, estudos têm sugerido que vítimas de maus-tratos infantis são também mais propensas a desenvolver comportamentos sexuais de risco (CSR) no final da adolescência ou na idade adulta, entre eles ter múltiplos parceiros sexuais, expor-se a relações sexuais desprotegidas e comércio sexual ${ }^{7-9}$, como forma de aliviar os sintomas emocionais ou, ainda, como consequência do abuso de substâncias ${ }^{10}$.

Há, ainda, evidência de que os efeitos a longo prazo dos maus-tratos infantis podem variar de acordo com o sexo ${ }^{7,11-15}$. Contudo, os resultados encontrados são inconclusivos, visto que alguns estudos sugerem que o risco, para alguns desfechos sexuais de risco, é maior em mulheres ${ }^{14,15}$, enquanto outros mostram que eles afetam mais os homens ${ }^{13}$.

Uma revisão sistemática e metanálise realizada, avaliando apenas a exposição ao abuso sexual $^{16}$, concluiu que o abuso sexual é um fator de risco significativo para a ocorrência de CSR e encontrou que a magnitude da associação foi semelhante para homens e mulheres. Apesar disso, ainda há inconsistência da evidência relativa a outras formas de maus-tratos infantis, como negligência e abuso físico, que são até mais prevalentes que o abuso sexual.

Os estudos existentes apresentam algumas limitações. A maior parte deles avalia apenas a população feminina ${ }^{8,17,18}$ e utiliza o abuso sexual como o único indicador de maus-tratos ${ }^{7,16,19}$.

Esta revisão sistemática teve o intuito de identificar os estudos que avaliaram a associação entre diferentes tipos de maus-tratos infantis e comportamento sexual de risco na idade adulta.

\section{Métodos}

Nesta revisão, a exposição aos maus-tratos infantis foi definida da seguinte forma:

Abuso sexual: envolvimento de uma criança em atividade sexual que ela não compreende completamente ou para a qual não está preparada, ou que viola as leis ou os tabus sociais da criança e da sociedade ${ }^{20}$.

Abuso físico: uso intencional de força física contra a criança e que resulta ou tem uma alta probabilidade de causar dano à sua saúde, sobrevivência, desenvolvimento ou dignidade. Compreende também bater, chutar, sacudir, morder, estrangular, queimar, envenenar e sufocar ${ }^{20}$.

Abuso emocional e psicológico: falha ao longo do tempo por parte de um dos pais ou cuidador, ou ambos, em fornecer um ambiente apropriado para o desenvolvimento da criança. São exemplos de abuso emocional e psicológico: restrição de movimento, menosprezo, culpar, ameaçar, assustar, discriminar ou ridicularizar e outras formas não físicas de rejeição ou tratamento hostil ${ }^{20}$.

Negligência: falha ao longo do tempo por parte dos pais ou da família em prover o desenvolvimento e bem-estar da criança em uma ou mais das seguintes áreas - saúde, educação, desenvolvimento emocional, nutrição, abrigo e condições seguras de vida ${ }^{20}$.

Violência doméstica: vivenciar ou testemunhar brigas com agressão física em casa entre adultos ou um adulto que agrediu uma criança ou um adolescente.

Comportamentos sexuais de risco: comportamentos sexuais que aumentam o risco de contrair o vírus da imunodeficiência humana (HIV) e outras infecções sexualmente transmissíveis (ISTs), como ter início sexual precoce, relações sexuais desprotegidas, ter tido múltiplos parceiros sexuais e se envolver em comércio sexual e prostituição.

\section{Estratégias de busca}

A revisão sistemática da literatura foi registrada no PROSPERO, banco internacional de revisões sistemáticas registradas prospectivamente e realizada de acordo com os itens propostos pelo PRISMA (Principais Itens para Relatar Revisões sistemáticas e Meta-análises). A busca bibliográfica foi feita nas bases eletrônicas U.S. National Library of Medicine and the National Institutes Health (PubMed), Web of Science, American Psichological Association (APA PsycNET) e da Literatura Latino-Americana e do Caribe em Ciên- 
cias da Saúde (Lilacs). Foram usados os seguintes descritores: ("adverse childhood experiences" OR "child sexual abuse" OR "abuse child*" OR "child* mistreatment" OR "child* maltreatment" OR "child* neglect" OR "child ${ }^{\star}$ abuse" OR "child* physical abuse" OR "abuse, physical" OR "physical violence" OR "physical maltreatment" OR "domestic violence" OR "family violence" OR "child* adversity" OR maltreatment OR neglect OR "parental violence" OR "physical abuse" OR violence OR "violence against children" OR "sex offenses" OR "sexual abuse" OR "sexual assault") AND ("sexual behavior" OR "unsafe sex" OR "sexual health" OR "sexually transmitted diseases" OR "sexually transmitted infections" OR “sexual partners" OR “risk-taking” OR "sexual outcomes" OR "risky sexual behavior" OR “early onset sexual activity" OR "sexual risk taking” OR "sexual risk behaviors" OR harass). Foi utilizado um operador * em "child*" para capturar possíveis variações nos termos "child", “children" ou "childhood".

As buscas foram realizadas sem restrição de idiomas e período. No entanto, apenas artigos publicados em português, inglês e espanhol foram incluídos. As bases de dados foram consultadas entre 25 de fevereiro e 31 de março de 2019. Os artigos foram importados para o software Endnote.

\section{Critérios de inclusão e exclusão}

Foram incluídos artigos originais que: (i) possuíam delineamento transversal, coorte ou caso-controle, não necessariamente de base populacional; (ii) avaliaram indivíduos que foram expostos a maus-tratos (abuso sexual, abuso físico, violência doméstica, abuso emocional e/ou negligência) até os 18 anos; (iii) avaliaram CSR na idade adulta (início sexual precoce, uso inconsistente de preservativo ou relação sexual desprotegida, promiscuidade, prostituição/comércio sexual e múltiplos parceiros sexuais).

Foram excluídos os estudos: (i) que se tratavam de teses, dissertações, cartas ao editor; (ii) realizados com amostras de diferentes faixas etárias (crianças, adolescentes e adultos) avaliando a exposição na vida adulta e que não reportaram a medida separada para infância/adolescência; (iii) que avaliaram outras experiências adversas em conjunto com os maus-tratos e não apresentaram a medida de associação isolada para os maus-tratos de interesse; (iv) conduzidos em populações com características muito específicas, que não são generalizáveis, como gestantes, indivíduos portadores de doenças como HIV ou outras ISTs, com problemas mentais e população em situação de "alto risco", como moradores de rua, usuários de drogas injetáveis ou que realizavam comércio sexual.

\section{Triagem e seleção}

Primeiro os trabalhos duplicados foram excluídos, depois os títulos dos artigos foram lidos e todos aqueles sem relação com o tema ( $\mathrm{n}=$ 18.658) foram excluídos. Após essa etapa, a partir dos resumos, foram selecionados os artigos que obedeciam aos critérios de inclusão. Dos artigos lidos na íntegra, selecionou-se os que atendiam a todos os critérios de inclusão. Os artigos incluídos ao final tiveram suas referências revisadas com o intuito de selecionar estudos não captados nas bases de dados acessadas. Nesse processo, mais três artigos foram adicionados ao estudo.

A Figura 1 demonstra o fluxograma completo do processo de seleção dos artigos que compõem essa revisão.

\section{Extração dos dados e avaliação da qualidade}

A extração dos dados foi realizada pela primeira autora, utilizando uma planilha previamente elaborada, compilando as seguintes informações: autor, ano, país do estudo, delineamento, tamanho amostral, população, tipo de exposição e de desfechos avaliados, variáveis utilizadas para ajuste, medida de efeito e intervalo de confiança. A avaliação da qualidade dos estudos indica a confiança que se terá nos resultados da revisão, que podem estar sistematicamente diferentes da realidade que se quer retratar devido a três principais fontes de viés: seleção, aferição e variáveis de confusão ${ }^{21}$.

Para avaliar a qualidade individual dos estudos e o risco de viés foi utilizada a Newcastle-Ottawa Scale ${ }^{22}$ para estudos longitudinais, de coorte e caso-controle. A escala contempla os seguintes domínios de avaliação do risco de viés: seleção dos pacientes (generalização e aplicabilidade), comparabilidade dos grupos no estudo, métodos para avaliação dos desfechos (estudos de coorte), definição da exposição (caso-controle) e acompanhamento adequado. Ela também fornece uma pontuação de classificação em que itens que apresentam alta qualidade recebem uma estrela, e cada estudo pode ter no máximo nove estrelas. Foi utilizada também uma versão adaptada da Newcastle-Ottawa Scale para avaliar estudos com delineamento transversal ${ }^{23}$. 


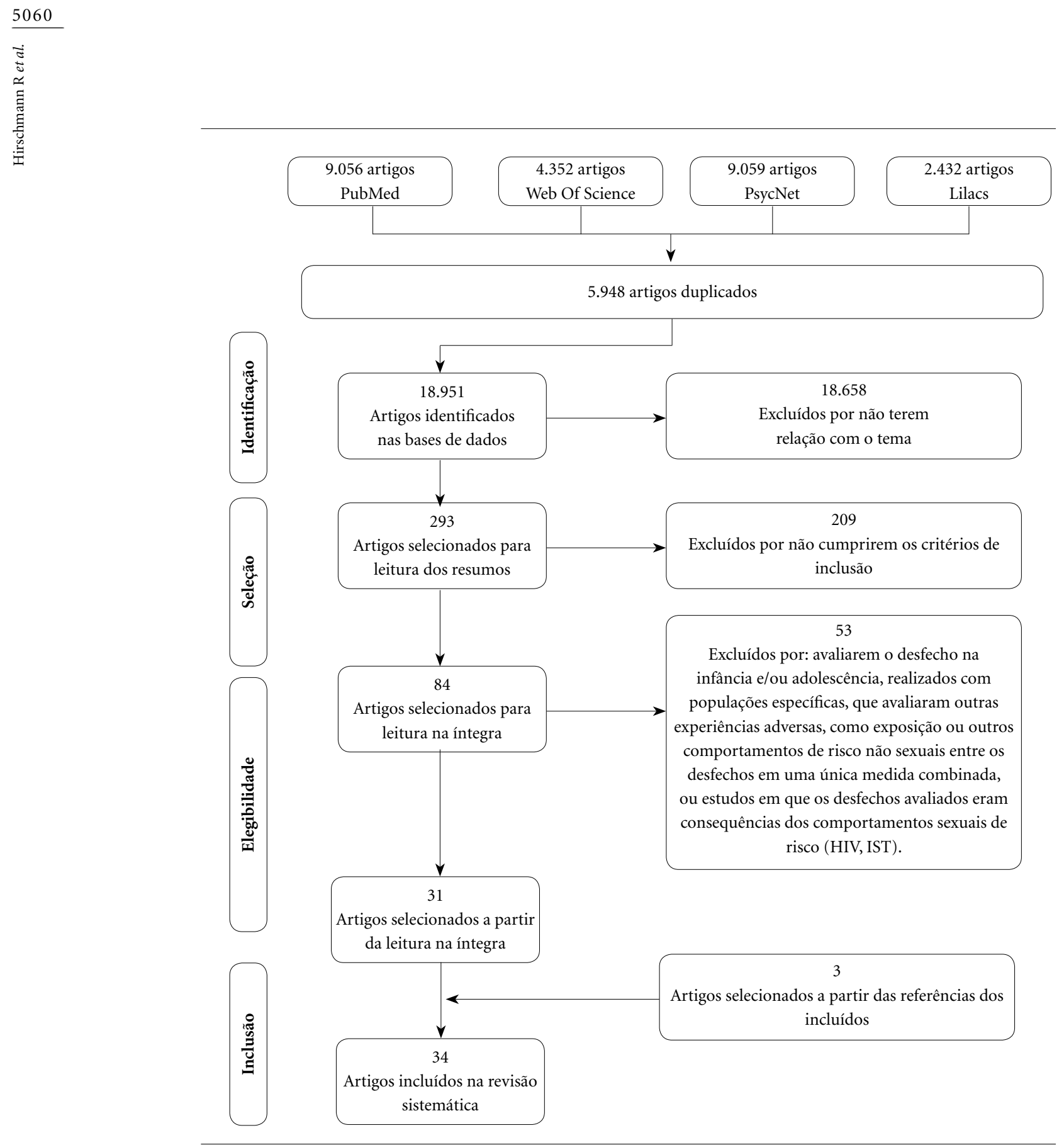

Figura 1. Fluxograma de seleção dos artigos.

Fonte: Elaborado pelos autores.

A avaliação da qualidade dos estudos, de acordo com as escalas utilizadas, está descrita em https://doi.org/10.48331/scielodata.YPXI29. $\mathrm{O}$ único artigo caso-controle ${ }^{13}$ encontrado não pôde ser avaliado com a escala ${ }^{22}$, uma vez que não apresenta metodologia clara o suficiente.

A revisão, em todo seu processo, foi realizada por duas autoras de forma independente $(\mathrm{RH}$ e RCM). As discordâncias foram resolvidas por consenso entre o par e, quando necessário, incluiu a avaliação de uma terceira pessoa.

\section{Identificação e seleção dos artigos incluídos}

Foram encontrados um total de 24.899 estudos nas bases de dados acessadas PubMed, Web Of Science, PsycNET e Lilacs, dos quais 5.948 estavam duplicados. Os 18.951 estudos restantes 


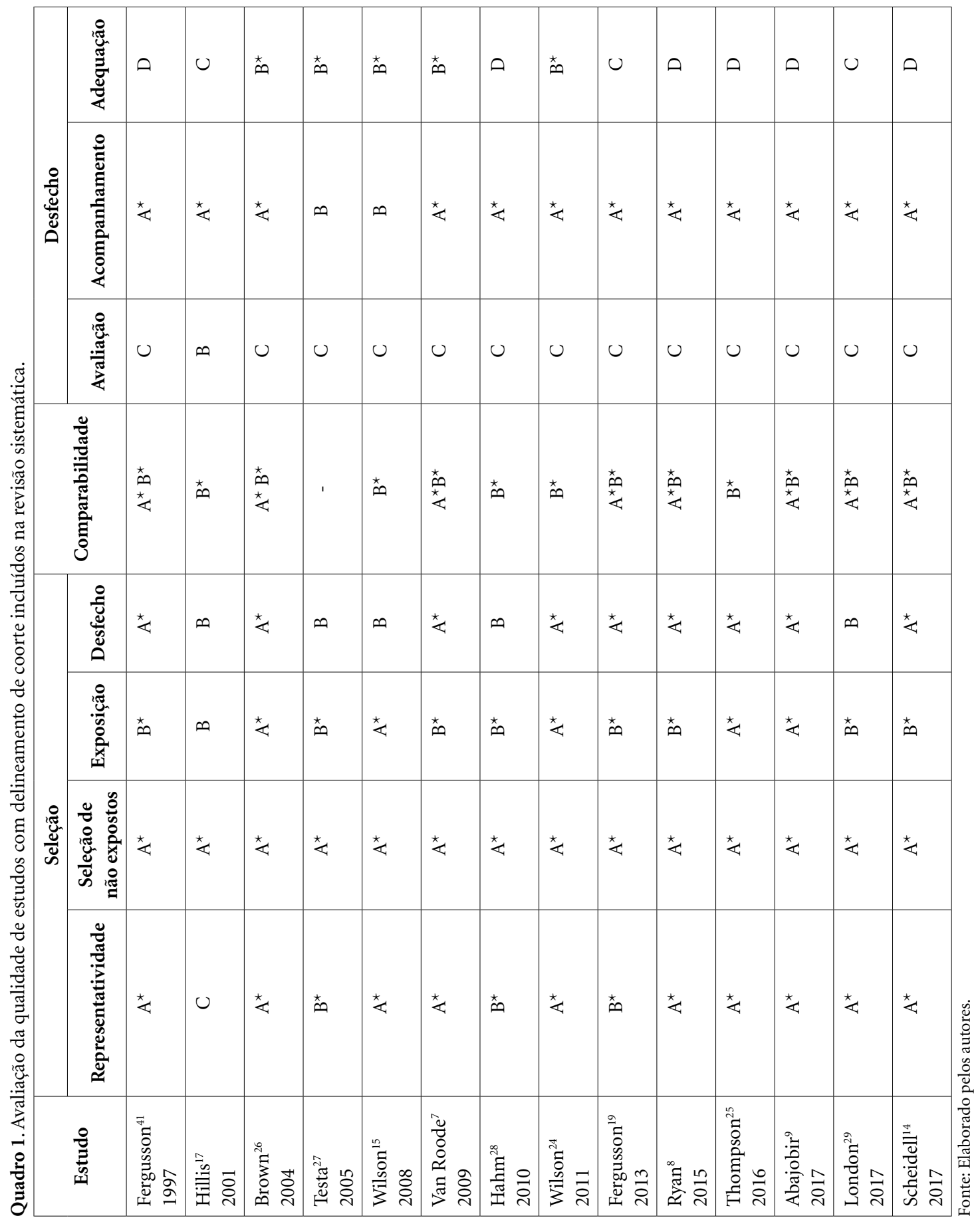

tiveram seus títulos lidos. Desses, selecionou-se 293 artigos para a leitura dos resumos e os outros 18.658 foram excluídos devido ao não cumprimento dos critérios preliminares de elegibilidade. Dos 84 artigos selecionados a partir dos resumos, 53 foram excluídos por descumprirem os critérios de elegibilidade, com base em leitura concisa dos artigos completos.

Dessa forma, 31 estudos foram selecionados. Somados a estes, encontramos mais três artigos nas referências dos escolhidos, totalizando ao final 34 estudos incluídos para revisão (Figura 1). 
Quadro 2. Avaliação da qualidade de estudos com delineamento transversal incluídos na revisão sistemática

\begin{tabular}{|c|c|c|c|c|c|c|c|}
\hline \multirow[b]{2}{*}{ Estudo } & \multicolumn{4}{|c|}{ Seleção } & \multirow[b]{2}{*}{ Comparabilidade } & \multicolumn{2}{|c|}{ Desfecho } \\
\hline & Representatividade & $\begin{array}{c}\text { Tamanho } \\
\text { da amostra }\end{array}$ & $\begin{array}{c}\text { Não } \\
\text { respondentes }\end{array}$ & Exposição & & Avaliação & $\begin{array}{c}\text { Teste } \\
\text { estatístico }\end{array}$ \\
\hline $\begin{array}{l}\text { Zieler }^{30} \\
1991\end{array}$ & $\mathrm{~A}^{*}$ & B & B & $\mathrm{C}$ & B & $\mathrm{C}^{*}$ & $A^{*}$ \\
\hline $\begin{array}{l}\text { Batten }^{31} \\
2001\end{array}$ & $\mathrm{D}$ & B & $\mathrm{C}$ & $\mathrm{C}$ & B & $\mathrm{B}^{* *}$ & B \\
\hline $\begin{array}{l}\text { Holmes }^{32} \\
2005\end{array}$ & $A^{*}$ & B & B & $\mathrm{C}$ & $A^{*}$ & $\mathrm{C}^{*}$ & $A^{*}$ \\
\hline $\begin{array}{l}\text { Holmes }^{33} \\
2005\end{array}$ & $A^{*}$ & B & $\mathrm{C}$ & $\mathrm{C}$ & B & $\mathrm{C}^{*}$ & $A^{*}$ \\
\hline $\begin{array}{l}\text { Steel }^{42} \\
2005\end{array}$ & $\mathrm{~A}^{*}$ & $\mathrm{~A}^{*}$ & $A^{*}$ & C & $\mathrm{B}$ & $\mathrm{C}^{*}$ & $A^{*}$ \\
\hline $\begin{array}{l}\text { Randolph } \\
2006\end{array}$ & $\mathrm{C}$ & B & $\mathrm{C}$ & $\mathrm{C}$ & $\mathrm{B}$ & $\mathrm{C}^{*}$ & B \\
\hline $\begin{array}{l}\text { Ramiro }^{43} \\
2010\end{array}$ & $\mathrm{~A}^{*}$ & $A^{*}$ & $A^{*}$ & $\mathrm{C}$ & $A^{*}$ & $\mathrm{~B}^{\star *}$ & $A^{*}$ \\
\hline $\begin{array}{l}\text { Roemmele }^{35} \\
2011\end{array}$ & $\mathrm{D}$ & $\mathrm{C}$ & $\mathrm{C}$ & $\mathrm{C}$ & B & $\mathrm{C}^{*}$ & $A^{*}$ \\
\hline $\begin{array}{l}\text { Hahm }^{18} \\
2012\end{array}$ & $A^{*}$ & B & $A^{*}$ & $\mathrm{C}$ & $A^{*}$ & $\mathrm{C}^{*}$ & $A^{*}$ \\
\hline $\begin{array}{l}\text { Lacelle }^{44} \\
2012\end{array}$ & $\mathrm{~A}^{*}$ & $\mathrm{C}$ & $\mathrm{C}$ & $\mathrm{C}$ & $\mathrm{B}$ & $\mathrm{C}^{*}$ & $A^{*}$ \\
\hline $\begin{array}{l}\text { Peltzer }{ }^{47} \\
2012\end{array}$ & $\mathrm{~A}^{*}$ & $\mathrm{C}$ & $\mathrm{C}$ & $\mathrm{C}$ & $A^{*}$ & $\mathrm{C}^{*}$ & B \\
\hline $\begin{array}{l}\text { Watson }^{36} \\
2013\end{array}$ & $\mathrm{C}$ & $\mathrm{C}$ & $\mathrm{C}$ & $\mathrm{C}$ & $\mathrm{B}$ & $\mathrm{C}^{\star}$ & B \\
\hline $\begin{array}{l}\text { Richter }^{48} \\
2014\end{array}$ & $A^{*}$ & B & $\mathrm{C}$ & $\mathrm{C}$ & $A^{*}$ & $\mathrm{C}^{*}$ & $\mathrm{~A}^{*}$ \\
\hline $\begin{array}{l}\text { Walsh }^{37} \\
2014\end{array}$ & $\mathrm{~A}^{*}$ & B & C & C & B & $\mathrm{C}^{*}$ & $\mathrm{~A}^{*}$ \\
\hline $\begin{array}{l}\text { Oshri }^{38} \\
2014\end{array}$ & C & C & C & C & B & $C^{*}$ & B \\
\hline $\begin{array}{l}\text { Bogolyubova }^{45} \\
2016\end{array}$ & $\mathrm{~A}^{*}$ & B & C & C & $\mathrm{A}^{*}$ & $\mathrm{C}^{*}$ & $\mathrm{~A}^{*}$ \\
\hline $\begin{array}{l}\text { Kogan }^{39} \\
2016\end{array}$ & B & B & C & C & $B$ & $C^{*}$ & B \\
\hline $\begin{array}{l}\mathrm{Hahm}^{40} \\
2017\end{array}$ & $\mathrm{~A}^{*}$ & B & C & C & $A^{*}$ & $\mathrm{C}^{\star}$ & $A^{*}$ \\
\hline $\begin{array}{l}\text { Tang }^{46} \\
2018\end{array}$ & $\mathrm{~A}^{*}$ & B & B & C & $\mathrm{A}^{*}$ & $C^{\star}$ & $\mathrm{A}^{*}$ \\
\hline
\end{tabular}

Fonte: Elaborado pelos autores.

Os seguintes motivos, de maneira geral, fizeram com que estudos não cumprissem os critérios de elegibilidade para inclusão: (i) avaliaram os desfechos ao longo da vida, incluindo infância ou adolescência; (ii) realizados em populações específicas, como usuários de drogas, pacientes atendidos em clínicas para ISTs, população selecionada pela orientação sexual, pacientes HIV positivo; (iii) avaliaram exposição ou desfechos como uma medida única que incluía outras for- 
mas de experiências vivenciadas e/ou comportamentos de risco distintos dos considerados nesta revisão; (iv) desfechos avaliados eram consequências dos CSR, como ISTs e HIV.

\section{Descrição dos estudos}

Foram identificados 34 estudos elegíveis, os quais estão resumidamente descritos em https:// doi.org/10.48331/scielodata.YPXI29. Foram publicados entre os anos de 1991 e 2018, em 12 países diferentes (Estados Unidos ${ }^{8,13-15,18,19,24-40}$, Nova Zelândia $^{7,17,41}$, Austrália ${ }^{9}$, Suécia ${ }^{42}$, Filipinas ${ }^{43}$, Canadá $^{44}$, Rússia ${ }^{45}$, China ${ }^{46}$, Costa do Marfim ${ }^{47}$, Zimbábue, Tanzânia e África do $\mathrm{Sul}^{48}$ ).

Os participantes dos estudos foram selecionados entre população geral7 7,9,19,26,32,33,41 , escolares ou universitários ${ }^{30,31,34-38,44-47}$, e casos registrados em atendimento de proteção infantil ${ }^{15,24-26}$. Doze estudos avaliaram amostras compostas apenas por mulheres ${ }^{8,17,18,27,28,31,34-36,40,41,44}$ e três incluíram somente indivíduos do sexo masculino ${ }^{32,33,39}$. Com relação aos tipos de estudos, 15 possuem delineamento longitudinal ${ }^{7-9,13-15,17,19,24-29,41}$ (14 coorte e um caso-controle) e 19 são transversais ${ }^{18,30-40,42-48}$.

Dos tipos de maus-tratos, o abuso sexual foi o mais avaliado entre os estu$\operatorname{dos}^{7-9,13-15,17-19,24-32,34-38,40-48}$, seguido por abuso físico $^{8,9,13,15,17,18,24-26,28,29,33,35,37,39,43,44,47,48}$ e negligência $^{8,9,13,15,18,24-26,28,29,39,43,45}$. Apenas três avaliaram a violência doméstica (testemunho) como exposição ${ }^{39,43,44}$. Dois estudos utilizaram somente uma medida combinada de dois ou mais maus-tratos em suas análises ${ }^{13,48}$.

Entre as exposições, em geral o abuso sexual foi avaliado como envolvimento em atividades sexuais indesejadas ou inapropriadas, toque indesejado, carícias, abraços ou beijos de uma maneira sexual, ou sexo forçado $9,15,17,24,27,41,46,47$. Alguns avaliaram os graus de severidade da exposição ao abuso, desde sexual sem contato até relações sexuais tentadas ou concluídas por via oral, vaginal ou anal ${ }^{19,41,46}$. Nem todos os artigos mencionavam quem cometeu o abuso, alguns utilizaram como critério qualquer adulto ou indivíduo mais velho ${ }^{26,32,43}$, outros questionavam a frequência de abuso sexual cometido pelos pais, cuidadores ou outros adultos ${ }^{8,14,17,18,28,29,40}$.

A exposição ao abuso sexual foi avaliada a partir do autorrelato ${ }^{7,8,14,17,19,26-29,30,32,34,41}$, da utilização de instrumentos para avaliação ${ }^{31,36-38,43,45}$, como o Wyatt Sexual History Questionnaire ${ }^{31}$, ou a partir de casos de registros oficiais ${ }^{15,24-26}$.

Considerou-se abuso físico ser machucado ou agredido fisicamente por um dos pais, parente, cuidador ou outra pessoa com que vivia $^{8,9,17,18,26,28,29,35,39,43}$, ameaças com arma ou faca $^{35,44}$, bem como casos de lesões, hematomas, feridas, cortes e lacerações ${ }^{15,24}$. Alguns foram definidos a partir de casos reportados pelo serviço de proteção à criança ${ }^{24-26}$. O uso de questionários para avaliar o abuso físico também foi observado, como o Conflict Tactics Scales (CTS) ${ }^{33,44}$ e o Childhood Trauma Questionnaire (CTQ) ${ }^{37}$.

Indivíduos foram identificados como expostos ao abuso emocional a partir de casos reportados por serviço de proteção à criança ${ }^{25}$, por terem sido submetidos a qualquer tipo de privação emocional ou trauma ${ }^{9,35}$ e ter sido insultado ou verbalmente ofendido ${ }^{29,43}$.

A negligência foi avaliada a partir de casos de registros oficiais dessa exposição ${ }^{15,24-26}$, definida como deficiência dos pais no cuidado infantil, no provimento das necessidades básicas e médicas ${ }^{8,9,18,28,29,39,43}$ e ser deixado sozinho sem a presença de um adulto ${ }^{18,28,29}$. Já a violência doméstica foi definida como a mãe ser tratada com violência $^{39,43}$, ou o testemunho de violência física e violência psicológica ${ }^{44}$.

Os desfechos entre os estudos foram avaliados de diferentes formas e em distintos períodos recordatórios, mas na maioria dos estudos os CSR avaliados incluíram idade de início sexual ou início sexual precoce antes dos 15 anos, sexo desprotegido na última relação sexual, múltiplos parceiros sexuais, sexo com desconhecidos, prostituição como receber dinheiro por sexo, comércio sexual, pagar ou ser pago por sexo, intenção de envolvimento em sexo de risco e sexo sob a influência de consumo de álcool ou outras drogas.

Os fatores de confusão mais utilizados como variáveis de ajuste nos estudos foram sexo, idade, etnia, quociente de inteligência, nível socioeconômico, idade materna na primeira gravidez, educação materna, situação conjugal dos pais, medidas de apoio social e funcionamento familiar (conflitos conjugais dos pais, separação parental, histórico parental de problemas com criminalidade ou uso de substâncias, mudança de um dos pais (até 15 anos), assim como problemas de internalização, sintomas depressivos, evasão escolar e ocorrência de maus-tratos infantis, excluindo a exposição de interesse. Outras características, como ter um parceiro sexual estável, ter parceiro do mesmo sexo, número de parceiros sexuais e envolvimento em comércio sexual, também foram analisadas como fatores de confusão.

Onze estudos avaliaram possíveis mediadores da relação entre maus-tratos infantis e $\mathrm{CSR}^{8,24,25,27,32,34,35-39}$. Desses, quatro possuíam deli- 
neamento longitudinal ${ }^{8,24,25,27}$ e sete eram transversais $^{32,34,35,37-39}$. Os estudos exploraram diferentes mediadores dessa relação, entre eles: sintomas afetivos, transtorno de estresse pós-traumático, relacionamentos de risco, esquemas relacionais defensivos e desadaptativos precoces (desconexão/rejeição, uso de drogas e álcool, comportamento criminoso e delinquente), autoeficácia sexual, vigilância corporal, desenvolvimento puberal, características do parceiro e satisfação no relacionamento, abuso sexual adulto e atitudes sexuais permissivas.

\section{Resultados}

Os 34 estudos revisados estão resumidamente apresentados nas tabelas 1 e 2 de acordo com a exposição de interesse. Os resultados são apresentados na forma de odds ratio (OR), com intervalos de confiança de 95\% (IC 95\%), ou coeficiente beta e valor p quando disponível.

\section{Abuso sexual e comportamentos sexuais de risco}

Abuso sexual foi o tipo de maus-tratos mais avaliado entre os estudos, sendo reportado em 32 artigos dos 34 incluídos nesta revisão (Tabela 2).

De modo geral, as pesquisas observaram que o abuso sexual infantil ( $<18$ anos) tem relação com CSR na idade adulta (18 a 92 anos). Mais especificamente, entre os resultados dos estudos longitudinais, observou-se que indivíduos que sofreram abuso sexual relataram que se envolveram em comportamentos sexuais de maior risco, como início sexual mais precoce $e^{9,15,17,19,26,27,41}$, maior número de parceiros sexuais ao longo da vida $^{7,8,14,17,19,27,29}$ e comércio sexual ${ }^{14,29}$, quando comparados àqueles que não sofreram abusos. Três estudos com esse delineamento ${ }^{24,25,28}$ não encontraram qualquer associação entre abuso sexual e múltiplos parceiros sexuais, idade de início sexual e comércio sexual, objetivo de suas análises. Entre os estudos transversais, os resultados variaram. Alguns encontraram associação entre abuso sexual e os desfechos citados, como início sexual precoce $^{42,43}$, sexo desprotegido ${ }^{31,38,40,43,45}$, múltiplos parceiros sexuais ${ }^{32,34,35,40,43,46}$, escore de $\operatorname{CSR}^{36,44}$ e prostituição $\mathrm{O}^{30,42}$. Um dos estudos encontrou essa associação apenas entre as formas mais graves do abuso (com penetração) ${ }^{46}$. Dos 16 estudos transversais que avaliaram essa relação, cinco não encontraram associação entre abuso sexual e tais desfechos ${ }^{18,30,31,37,47}$.
A medida de efeito (OR) da relação entre abuso sexual e início sexual precoce variou de 0,8 (IC 95\%: 0,4-1,4) a 11,6 (IC 95\%: 6,3-21,4). Para o desfecho múltiplos parceiros sexuais o OR variou de 1,2 (IC 95\%: 0,6-2,4) a 3,6 (IC 95\%: 2,7-5,0) e para relação sexual desprotegida foram encontradas medidas de efeito entre 1,0 (IC 95\%: 0,7-1,2) e 4,3 (IC 95\%: 2,0-9,2). Para os desfechos prostituição, sexo por dinheiro ou comércio sexual o OR variou de 1,0 (IC 95\%: 0,1-7,7) a 6,0 (IC 95\%: 0,83-43,71).

\section{Abuso físico e comportamentos sexuais de risco}

Dezessete estudos avaliaram abuso físico, no entanto grande parte deles encontrou fraca associação ou sua ausência ${ }^{18,24,26,33,39,43,47}$ na relação entre abuso físico e pelo menos um tipo de CSR. Em oito estudos a relação esteve associada aos seguintes CSR: início sexual precoce $e^{9,15,17,28}$, múltiplos parceiros sexuais ${ }^{17,28,35}$, comércio sexual/ prostituição ${ }^{15,24,29}$ ou escore de comportamento sexuais de risco ${ }^{44}$ (Tabela 2).

A medida de efeito (OR) da relação entre abuso físico e início sexual precoce variou de 1,0 (IC 95\%: 0,4-2,7) a 2,6 (IC 95\%: 1,35-3,13). Para o desfecho múltiplos parceiros sexuais, o OR variou de 0,91 (IC 95\%: 0,72-1,16) a 4,53 (IC 95\%: 0,63-32,6). Nessa última medida de efeito, o desfecho foi o uso inconsistente de preservativo e múltiplos parceiros sexuais, avaliados de maneira combinada. Já quanto ao uso inconsistente de preservativo isoladamente, este foi avaliado apenas em um estudo ${ }^{47}$, que mostrou somente as medidas separadas por sexo, a OR para os homens foi de 0,96 (IC 95\%: 0,54-1,72), e para mulheres de 1,42 (IC 95\%: 0,41-2,57). Para os desfechos prostituição, comércio sexual ou sexo por dinheiro, a OR variou de 0,89 (IC 95\%: 0,42$1,90)$ a 3,35 (IC 95\%: 1,38-8,15).

\section{Abuso emocional e comportamentos sexuais de risco}

O abuso emocional foi classificado de distintas formas, dificultando a comparação entre os estudos. A relação entre abuso emocional e comportamentos sexuais ainda não é clara, visto que poucas pesquisas avaliam essa exposição de maneira independente $e^{9,25,29,35,43}$. Três possuem delineamento longitudinal ${ }^{9,25,29}$ e dois são transversais $^{35,43}$, no entanto não há consistência entre os resultados encontrados. Apenas um estudo longitudinal encontrou associação entre abuso 
emocional e início sexual precoce $(<15 \text { anos })^{9}$, não sendo encontrada em outro estudo, transversal, que avaliou esse mesmo desfecho ${ }^{43}$.

Aqueles que sofreram abuso emocional antes dos 18 anos apresentaram maior chance de ter múltiplos parceiros sexuais na vida adulta. Três dos cinco estudos que avaliaram essa relação, um com delineamento longitudinal ${ }^{29}$ e outros dois transversais ${ }^{35,43}$, encontraram associação estatisticamente significativa ${ }^{29,43}$. Para outros desfechos de CSR, como envolvimento com comércio se$\mathrm{xual}^{25} \mathrm{e} / \mathrm{ou}$ sexo desprotegido ${ }^{29}$ na idade adulta, não foi encontrada associação significativa ${ }^{25,29}$ (Tabela 2).

A medida de efeito da relação entre abuso emocional e início sexual precoce variou de 1,1 (IC 95\%: 0,7-1,8) a 2,15 (IC 95\%: 1,27-3,65). Para o desfecho múltiplos parceiros sexuais, a OR variou de 0,96 (IC 95\%: 0,79-1,16) a 2,1 (IC 95\%: $1,5-3,0)$.

\section{Negligência e comportamentos sexuais de risco}

Essa exposição foi considerada em 11 estu$\operatorname{dos}^{8,9,15,18,24-26,28,29,39,43}$, sendo encontrada associação positiva com CSR e início sexual precoce ${ }^{9,15,28}$, prostituição ${ }^{15}$, comércio sexual ${ }^{29}$, múltiplos parceiros sexuais $^{9,24,28,29}$ e sexo desprotegido ${ }^{24,25}$ naqueles com delineamento longitudinal. Os estudos que obtiveram resultados estratificados por sexo encontraram que as mulheres apresentam maior medida de efeito para comportamentos como prostituição e comércio sexual quando comparadas aos homens ${ }^{15,29}$.

Entre os três estudos transversais encontra$\operatorname{dos}^{18,39,43}$, dois verificaram associação entre negligência e desfechos sexuais de risco ${ }^{39,43}$.

Apenas um avaliou as duas formas de negligência, física e psicológica, e a segunda esteve associada a início sexual mais precoce e múltiplos parceiros sexuais ${ }^{43}$. O mesmo estudo encontrou associação de negligência física apenas com múltiplos parceiros sexuais ${ }^{43}$. Em outra pesquisa, a negligência esteve positivamente associada a uma medida combinada dos desfechos, como número de parceiros sexuais, uso inconsistente de preservativo e/ou sexo sob influência de álcool ou dro$\operatorname{gas}^{39}$ (Tabela 2).

\section{Violência doméstica}

A exposição à violência doméstica foi acessada somente em três estudos, com delineamento transversal. Um deles avaliou a associação entre testemunho de violência psicológica ou física entre pais ou responsáveis e um escore de CSR que considerou início sexual antes dos 16 anos, diagnóstico médico de IST, uso inconsistente de preservativo na última relação com um novo parceiro e/ou ter usado drogas ou álcool durante atividade sexual no último ano, porém a violência doméstica, avaliada por meio do testemunho de violência física ou psicológica, não foi um preditor significativo de $\mathrm{CSR}^{44}$.

Nos outros dois estudos ${ }^{39,43}$, a violência doméstica foi avaliada como ter testemunhado a mãe ou madrasta ser tratada com violência. Uma medida combinada de CSR, início sexual precoce ou múltiplos parceiros sexuais foram avaliados como CSR, no entanto foi encontrada associação positiva apenas em um dos estudos com múltiplos parceiros sexuais ${ }^{43}$.

\section{Discussão}

A presente revisão sistemática identificou estudos que avaliaram a relação entre maus-tratos infantis e CSR, a maior parte em países com alta renda, como os Estados Unidos, e delineamento transversal. Menos estudos foram conduzidos em países mais pobres. A pobreza, conflitos familiares, estímulo cognitivo inadequado e problemas sociais como menor oportunidade de aprendizado servem como fatores combinados que podem estar interligados, fazendo com que as crianças residentes nos países com menor renda estejam mais expostas a eventos negativos, impedindo que atinjam seu potencial máximo ${ }^{43}$. Ainda é insuficiente, para conclusões mais precisas, o que se sabe sobre o efeito particular em longo prazo de algumas formas de maus-tratos menos avaliadas, como negligência, abuso emocional e violência doméstica sobre os CSR.

Observou-se, ainda, que entre os estudos há formas distintas de avaliar e conceituar os maustratos infantis enquanto exposição, entre eles o abuso sexual. Alguns pesquisadores o avaliaram como o indivíduo ser forçado à nudez ou ao toque sexual, outros como relação sexual concluída sem consentimento e envolvendo penetração. Isso tornou a tarefa de comparar os resultados dos estudos inviável.

Além disso, grande parte dos estudos incluídos nesta revisão avaliou somente a exposição ao abuso sexual7,14,19,27,30-32,34,36,38,40-42,46 ou amostras compostas apenas por mulheres $^{8,17,18,27,28,31,34,35,36,40,41,44}$, e seus resultados demonstram relação de longo prazo desse tipo de 
abuso e CSR. Os achados sugerem que o abuso sexual está diretamente relacionado ao início sexual precoce ${ }^{9,15,17,19,26,27,41}$, a ter múltiplos parceiros sexuais $7,8,14,17,19,27,29$, a fazer sexo desprotegido $^{38,41,45,46}$ e ao comércio sexual ${ }^{14,29}$. Esses comportamentos representam um fator de risco significativo para outras complicações de saúde, como ISTs e HIV, denotando a importância de se agir logo que ocorram, a fim de prevenir desfechos futuros. Há menos evidências publicadas acerca das consequências de maus-tratos na infância, como o abuso emocional e a negligência sobre o comportamento sexual. Novos estudos poderão responder a respeito desse tema.

As limitações notadas entre os estudos incluídos nesta revisão devem ser consideradas. Uma parcela deles possui amostras pequenas $^{13,18,30-34,38,39,41}$ e/ou selecionadas ao acaso ${ }^{32,33,42}$. É importante mencionar que, apesar de não ter tanta influência nos resultados de associação, pode afetar a medida de ocorrência dos estudos. Percebe-se também a ausência de uma definição conceitual mais ampla, abrangendo mais abusos e/ou negligência - como já mencionado, a falta de investigação do sexo como um modificador de efeito é uma limitação a ser ponderada, que deve ser mais explorada para o melhor entendimento de como se dá essa relação de acordo com o sexo. Além disso, há de se considerar que a forma de coleta das exposições pode ter influenciado nos resultados. Seis estudos ${ }^{13,15,24-26,29}$ utilizaram pelo menos uma medida de exposição de fontes secundárias (serviços de proteção à criança), que podem estar sujeitas à subnotificação ou ao sub -registro. Outros estudos acessaram, de maneira retrospectiva, as informações sobre a ocorrência dos maus-tratos, alguns na idade adulta, forma que pode levar ao sub-relato, reduzindo a medida de efeito, se sujeita a viés de memória. No entanto, por se tratar de eventos fora do cotidiano, e em geral com grande impacto, esse problema pode estar minimizado.

Entre os estudos publicados, poucos foram realizados com amostras de base populacional, uma vez que grande parte dos estudos avalia universitários, escolares, amostras selecionadas ao acaso ou a partir de casos de registros oficiais.
Sabe-se que a síntese de dados derivados de diferentes populações em estudo aumenta a probabilidade de viés e afeta a generalização e comparabilidade dos resultados. Contudo, a exclusão de estudos que avaliaram populações muito específicas, como um dos critérios de seleção, procurou amenizar este problema.

Nos resultados desse estudo, deve-se considerar ainda o viés de publicação, uma vez que alguns estudos podem não ter publicado os resultados de todos os desfechos planejados inicialmente, por não terem encontrado associação ou o resultado esperado.

Uma questão importante para entender os mecanismos da relação entre maus-tratos infantis e CSR nesse tipo de pesquisa é a análise de mediação, explorada em poucos desses estudos, a maioria deles com delineamento transversal, que não suportam inferências causais.

Os esforços para prevenir ou remediar CSR em adultos devem considerar a possível ocorrência de maus-tratos durante a infância, bem como desenvolver estratégias para a prevenção de abusos e negligências que envolvam professores, pais e/ou responsáveis como alvos principais de orientação de como proceder na ocorrência desses casos, incluindo também os profissionais de saúde, para que os mesmos recebam apoio no diagnóstico, registro e notificação desses casos de maus-tratos infantis. Para ajudar a modificar as consequências danosas dos maus-tratos ao longo da vida dos indivíduos, é fundamental que se construa um processo de diálogo em que as crianças também possam ser ouvidas e orientadas quando em situações abusivas.

\section{Conclusão}

Os maus-tratos infantis influenciam de maneira negativa os comportamentos sexuais na vida adulta. Os CSR são desfechos menos explorados e devem ser melhor investigados em estudos longitudinais, que permitem avaliar os efeitos sobre os comportamentos sexuais ao longo da vida e entender melhor os possíveis mecanismos envolvidos nessa relação. 


\section{Colaboradores}

As três autoras participaram da concepção, do planejamento, da interpretação e redação do trabalho e/ou revisão crítica do manuscrito, que foi lido e aprovado por todas as autoras.

\section{Referências}

1. World Health Organization (WHO). Child Maltreatment; 2018. [acessado 2019 Maio 23]. Disponível em: http://www.who.int/news-room/fact-sheets/detail/ child-maltreatment

2. Stoltenborgh M. The prevalence of child maltreatment across the globe: review of a series of meta-analyses. Child Abuse Review 2015; 24(1):37-50.

3. Gilbert R, Widom CS, Browne K, Fergusson D, Webb E, Janson S. Burden and consequences of child maltreatment in high-income countries. Lancet 2009; 373(9657):68-81.

4. Thornberry TP, Ireland TO, Smith CA. The importance of timing: the varying impact of childhood and adolescent maltreatment on multiple problem outcomes. Dev Psychopathol 2001; 13(4):957-979.

5. Green J, McLaughlin KA, Berglund PA, Gruber MJ, Sampson NA, Zaslavsky AM, Kessler RC. Childhood adversities and adult psychiatric disorders in the $\mathrm{Na}$ tional Comorbidity Survey Replication I: associations with first onset of DSM-IV disorders. Arch Gen Psychiatry 2010; 67(2):113-123.

6. Geoffroy MC, Pinto PS, Li L, Power C. Child neglect and maltreatment and childhood-to-adulthood cognition and mental health in a prospective birth cohort. J Am Acad Child Adolesc Psychiatry 2016; 55(1):33-40.

7. van Roode T, Dickson N, Herbison P, Paul C. Child sexual abuse and persistence of risky sexual behaviors and negative sexual outcomes over adulthood: findings from a birth cohort. Child Abuse Negl 2009; 33(3):161-72.

8. Ryan RM, Mendle J, Markowitz AJ. Early childhood maltreatment and girls' sexual behavior: The mediating role of pubertal timing. J Adoles Health 2015; 57(3):342-347.

9. Abajobir AA, Kisely S, Williams G, Stratherarn L, Najman JM. Risky sexual behaviors and pregnancy outcomes in young adulthood following substantiated childhood maltreatment: findings from aprospective birth cohort study. J Sex Res 2016; 55(1):106-119.

10. Littleton HL, Grills-Taquechel AE, Buck KS, Rosman L, Dodd JC. Health risk behavior and sexual assault among ethnically diverse women. Psychol Women $Q$ 2013; 37(1):7-21.

\section{Financiamento}

O presente trabalho foi realizado com apoio da Coordenação de Aperfeiçoamento de Pessoal de Nível Superior (CAPES).
11. Haydon AA, Hussey JM, Halpern CT. Childhood abuse and neglect and the risk of STDs in early adulthood. Perspect Sex Reprod Health 2011; 43(1):16-22.

12. Thibodeau M, Lavoie F, Hébert M, Blais M. Childhood maltreatment and adolescent sexual risk behaviors: unique, cumulative and interactive effects. Child Abuse Negl 2017; 72:411-420.

13. Negriff S, Schneiderman JU, Trickectt PK. Child maltreatment and sexual risk behavior: Maltreatment types and gender differences. J Dev Behav Pediatr 2015; 26(9):708-716.

14. Scheidell JD. Child sexual abuse and HIV-related substance use and sexual risk across the life course among males and females. J Child Sex Abus 2017; 26(5):519-534.

15. Wilson HW, Widom CS. An examination of risky sexual behavior and HIV in victims of child abuse and neglect: a 30-year follow-up. Health Psychol 2008; 27(2):149-158

16. Abajobir AA, Kisely S, Maravilha JC, Williams G, Najman JM. Gender differences in the association between childhood sexual abuse and risky sexual behaviors: a systematic review and meta-analysis. Child Abuse Negl 2016; 63:249-260.

17. Hillis SD, Anda RF, Felitti VJ, Marchbanks PA. Adverse Childhood Experiences and Sexual Risk Behaviors in Women: A Retrospective Cohort Study. Fam Plann Perspect 2001; 33(5):206-211.

18. Hahm HC. Do Asian-American women who were maltreated as children have a higher likelihood for HIV risk behaviors and adverse mental health outcomes? Womens Health Issues 2012; 22(1):35-43.

19. Fergusson DM, Mcleod GFH, Horwood LJ. Childhood sexual abuse and adult developmental outcomes: Findings from a 30-year longitudinal study in New Zealand. Child Abuse Negl 2013; 37(9):664-674.

20. World Health Organization (WHO). Preventing Child Maltreatment: a guide to taking action and generating evidence. Geneva: WHO/International Society for Prevention of Child Abuse and Neglect; 2006. 
21. Brasil. Ministério da Saúde (MS). Diretrizes metodológicas: elaboração de revisõe sitemática e metanálise de estudos observacionais comparative sobre fatores de risco e prognóstico. Brasilia: MS; 2014.

22. GA Wells, B Shea, D O'Connell, J Peterson, V Welch, M Losos, P Tugwell. The Newcastle-Ottawa Scale (NOS) for Assessing the Quality of Nonrandomized Studies in Meta-analyses. Ottawa Hospital Research Institute. [cited 2019 Out 13]. Available from: http://www.ohri. ca/programs/clinical_epidemiology/oxford.asp

23. Herzog R, Álvarez-Pasquin MJ, Díaz C, Del Barrio JL, Estrada JM, Gil Á. Are healthcare workers' intentions to vaccinate related to their knowledge, beliefs and attitudes? A systematic review. BMC Public Health 2013; 13:154.

24. Wilson HW, Widom CS. Pathways from childhood abuse and neglect to HIV-risk sexual behavior in middle adulthood. J Consult Clin Psychol 2011; 79(2):236246.

25. Thompson R, Lewis T, Neilson EC, English DJ, Litrownik AJ, Margolis B, Proctor L, Dubowitz H. Child maltreatment and risky sexual behavior: Indirect effects through trauma symptoms and substance use. Child Maltreatment 2016; 22.

26. Brown J, Cohen P, Chen H, Smailes E, Johnson JG. Sexual trajectories of abused and neglected youths. Dev Behav Pediatrics 2004; 25(2):77-82.

27. Testa M. Childhood sexual abuse, relationship satisfaction, and sexual risk taking in a community sample of women. J Consult Clin Psychol 2005; 73(6):11161124.

28. Hahm HC, Lee Y, Ozpnoff A, Van Wert M.J. The impact of multiple types of child maltreatment on subsequent risk behaviors among women during the transition from adolescence to young adulthood. $J$ Youth Adolescence 2010; 39;528-540.

29. London S, Quinn K, Scheidell JD, Frueh C, Khan MR. Adverse experiences in childhood and sexually transmitted infection risk from adolescence into adulthood. Sex Transm Dis 2017; 44(9):524-532.

30. Zierler S, Feingold L, Laufer D, Velentgas P, Kantrowitz-Gordon I, Mayer K. Adult survivors of childhood sexual abuse and subsequent risk of HIV infection. Am J Public Health 1991; 81(5):572-575.

31. Batten SV, Follete VM, Aban IB. Experiential avoidance and high-risk sexual behavior in survivors of child sexual abuse. J Child Sex Abus 2001; 10(2):101-120.

32. Holmes WC. Men's pathways to risky sexual behavior: role of co-occurring childhood sexual abuse, posttraumatic stress disorder, and depression histories. $J$ Urban Health 2005; 82(suppl. 1):89-99.

33. Holmes WC. Sammel MD. Brief communication: physical abuse of boys and possible associations with poor adult outcomes. Ann Intern Med 2005; 143(8):581-586.

34. Randolph ME, Mosack KE. Factors mediating the effects of childhood sexual abuse on risky sexual behavior among college women. J Psychology \& Human Sexuality 2006;18(1): 23-41.

35. Roemmele M, Messman-Moore TL. Child abuse, early maladaptive schemas, and risky sexual behavior in college women. J Child Sex Abus 2011; 20(3):264-283.

36. Watson LB. A model linking diverse women's child sexual abuse history with sexual risk taking. Psychol Women Q 2013;37(1):22-37.
37. Walsh K. Pathway from child sexual and physical abuse to risky sex among emerging adults: the role of trauma-related intrusions and alcohol problems. $J$ Adolesc Health 2014; 54(4): 442-448.

38. Oshri, A. Child maltreatment types and risk behaviors: associations with attachment style and emotion regulation dimensions. Personality and Individual Differences 2015;73: 127-133.

39. Kogan SM. The influence of childhood adversity on rural black men's sexual risk behavior. Ann Behav Med 2016; 50(6):813-822.

40. Hahm HC. The associations between forced sex and severe mental health, substance use, and HIV risk behaviors among Asian American women. Violence Against Women 2017; 23(6):671-691.

41. Fergusson DM, Horwood J, Lynskey MT. Childhood sexual abuse, adolescent sexual behaviors and sexual revictimization. Child Abuse Negl 1997; 21(8):789803.

42. Steel JL, Herlitz CA. The association between childhood and adolescent sexual abuse and proxies for sexual risk behavior: a random sample of the general population of Sweden. Child Abuse Negl 2005; 29(10):1141-1153.

43. Ramiro LS. Adverse childhood experiences (ACE) and health-risk behaviors among adults in a developing country setting. Child Abuse Negl 2010; 34(11):842855.

44. Lacelle C. Child sexual abuse and women's sexual health: the contribution of CSA severity and exposure to multiple forms of childhood victimization. J Child Sex Abus 2012; 21(5):571-592.

45. Bogolyubova O, Skochilov R, Smykalo L. Childhood victimization and HIV risk behaviors among university students in Saint-Petersburg, Russia. AIDS Care 2016; 28(12):1590-1594.

46. Tang K. Childhood sexual abuse, risky sexual behaviors and adverse reproductive health outcomes among Chinese college students. Child Abuse Negl 2018; 84:123-130.

47. Peltzer K, Mental health, childhood abuse and HIV sexual risk behaviour among university students in Ivory Coast. Ann Gen Psychiatry 2012; 12(1):18.

48. Richter L, Komárek A, Desmond C, Celentano D, Morin S, Sweat M, Chariyalertsak S, Chingono A, Gray G, Mbwambo J, Coates T. Reported physical and sexual abuse in childhood and adult HIV risk behaviour in three African countries: findings from Project Accept (HPTN-043). AIDS Behav 2014; 18(2):381-389.

Artigo apresentado em 06/07/2019

Aprovado em 07/02/2020

Versão final apresentada em 09/02/2020

Editores-chefes: Romeu Gomes, Antônio Augusto Moura da Silva 